\title{
Magnetic Properties of Magnetoplasmonic Crystals Based on Commercial Digital Discs
}

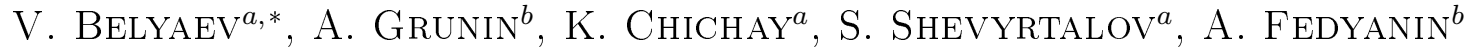 \\ AND V. RODIONOVA ${ }^{a, c}$
}

${ }^{a}$ Innovation Park and Institute of Physics and Technology, Immanuel Kant Baltic Federal University, Nevsky 14, 236041, Kaliningrad, Russia

${ }^{b}$ Lomonosov Moscow State University, Leninskie Gory 1, 119991, Moscow, Russia

${ }^{c}$ National University of Science and Technology MISiS, Leninsky 4, 119049, Moscow, Russia

Several topics of photonics are targeted at the enhancement of magneto-optical response in the nanostructures. One of the approaches is creating of the magnetoplasmonic crystals based on the substrates made of the digital discs (CD, DVD, BD) with ferromagnetic film. It is necessary to investigate in-plane anisotropy of magnetoplasmonic crystals and influence of the ferromagnetic layer thickness on increase of the mean free path of edge magnetoplasmons. Ni-based magnetoplasmonic crystals were investigated using the vibrating sample magnetometer by LakeShore. All samples had anisotropic in-plane magnetic properties because of the induced anisotropy caused by the stripe-like periodic structure of the substrates. A step-like behaviour of the hysteresis loops in case of the transverse plasmon propagation way was observed for Ni-based magnetoplasmonic crystals. Measurements along the plasmon propagation way showed near-rectangular hysteresis loops typical for the Ni-based thin films for certain samples. Step-like behaviour of the hysteresis loops for the Ni-based magnetoplasmonic crystals can be explained as a result of interaction between Ni films that covered the top and the lateral sides of the substrate stripes.

DOI: $10.12693 /$ APhysPolA.127.546

PACS: $75.70 .-\mathrm{i}, 75.30 . \mathrm{Gw}$

\section{Introduction}

Small values of the magneto-optical (MO) effects strongly restrict their practical applications and in recent years several topics of photonic researches were targeted at the development of new types of small and reliable MO-devices such as biosensors [1], optical nanoantennas [2], hybrid ultrafast nanophotonic devices for future telecommunications and data-recording [3]. Sensitivity of MO sensors can be enhanced by creating them on the base of the magnetophotonic crystals which allows one to enhance magneto-optical Faraday or Kerr effects at the edge of the photonic band gap or at the micro cavity mode [4]. Magnetoplasmonic nanostructures represent a special class of plasmonic nanostructures fabricated from magnetic metals (for example, from nickel, cobalt and iron or from composites of magnetic materials) and noble metals [5-8]. It was demonstrated that in such systems, it is possible both to enhance the MO activity of the system via the surface plasmon excitation, and to modulate the plasmon properties via application of the magnetic field [9].

Surface plasmon resonance (SPR) technique is an optical method of measurements of the refractive index of very thin layers of any material. When light is falling at defined angle it can interact with the delocalized electrons in the metal film creating plasmons and part of light energy is absorbed for excitation. The SPR technique

*corresponding author; e-mail: v.k.belyaev@gmail.com is based on the fact that, at certain conditions, surface plasmons on a metallic film can be excited by photons, thereby transforming a photon into a surface plasmon and it depends on the refractive index of the adsorbate [10]. Also the value of SPR depends on magnetic properties of magnetoplasmonic crystals (MPlCs) and can be influenced by changing of the material, thickness and continuity of the ferromagnetic layer, spatial profile of the substrate and the tension value. Changing of these parameters can influence upon magnetic properties as stronger as thinner the ferromagnetic layer.

SPR, as a surface oriented method, has shown a great potential for the affinity biosensors, allowing real-time analysis of the biospecific interactions without the use of the labeled molecules. The SPR sensor technology has been commercialized by several companies and has become a leading technology in the field of direct realtime observation of the biomolecular interactions [11].

Therefore, SPR method has already proved itself as a reliable method for sensor applications and we can suggest and develop a new type of the alternating magnetic field sensor based on the magnetoplasmonic crystal. Sensitivity of such sensors will depend on the value of the magnetic plasmon resonance which will depend on the MPlCs magnetic properties.

This article is devoted to investigations of magnetic properties of MPlCs structures for creating of a new type of the alternating magnetic field sensor. From the analysis of the MO response in zero magnetic field and in the external magnetic field of unknown magnitude we can get a value of magnitude of the external field with good accuracy. In such type of sensors the MO response of 
the MPlCs structure depends on the saturation state of the magnetic layer. This state depends on the external magnetic field. Therefore, first one should know the magnetic characteristics of the MPlCs. In this manuscript we present first results of investigation of MPlCs magnetic properties depending on thickness of layers and spatial profiles of substrates.

\section{Experiment details and samples}

MPlCs were fabricated by ion plasma deposition (IPD) of $\mathrm{Ni}$ on polymer templates of commercial digital discs. The polymer spiral gratings inside the Blu-ray disc (BD) and DVD have declared periods of 320 and $740 \mathrm{~nm}$, and profile heights of approximately 20 and $100 \mathrm{~nm}$, respectively. First, protective layer of the digital disc surface was carefully mechanically removed for saving spatial gratings intact. Then, metals (Ag or $\mathrm{Ni}$ ) were sputtered on the polymer gratings. In the end several structures were covered by $\mathrm{SiO}_{2}$ layer to prevent the oxidation. The structures without a protective layer have become covered by $\mathrm{Ni}_{2} \mathrm{O}$ (thickness of the layer is approximately $\approx 5 \mathrm{~nm}$ ), because of interaction with the atmosphere after fabrication. Detailed information about the fabricated samples can be found in Table.

\section{TABLE}

Composition of the fabricated samples.

\begin{tabular}{c|c|c|c}
\hline \hline No & Substrate & 1st layer [nm] & 2nd layer [nm] \\
\hline 1 & BD & $\mathrm{Ni}, 100$ & none \\
2 & DVD & $\mathrm{Ag}, 80$ & $\mathrm{Ni}, 10$ \\
3 & DVD & $\mathrm{Ni}, 100$ & $\mathrm{SiO}_{2}, 10$
\end{tabular}

We investigated magnetic properties of the MPlCs structures, based on the embossed substrates with different spatial profiles.

Magnetic properties of the fabricated samples were studied by vibrating sample magnetometer by LakeShore with maximum magnetic field of $12 \mathrm{kOe}$ and sensitivity up to $10^{-6}$ emu. All measurements were made at room temperature.

Spatial profiles of the polymer spiral gratings inside the substrates made of DVD and BD discs were obtained by the atomic-force microscopy (AFM).

\section{Results and discussion}

In-plane hysteresis loops were investigated for all fabricated structures. First result was in observation of induced anisotropy: we found axis of easy and hard magnetization for all Ni-based MPlCs structures. Easy magnetization axis was parallel to the direction of the substrate stripes while the hard magnetization axis was perpendicular to the direction of the substrate stripes. The direction of the substrate stripes is perpendicular to the plasmon propagation way. In all figures direction is specified relative to the direction of the substrate stripes. Figure 1 represents the results of in-plane measurements for the Ni-based MPIC on the substrate made of the BD digital disc (position 1 from Table) for different angles.

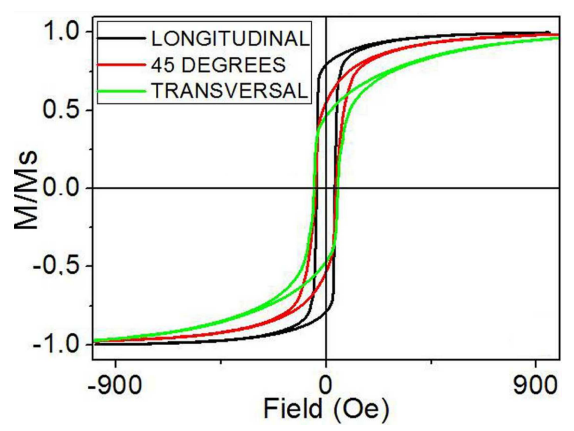

Fig. 1. Induced anisotropy for Ni-based MPlCs on BD substrate - $\mathrm{BD} / / \mathrm{Ni}(100)$.

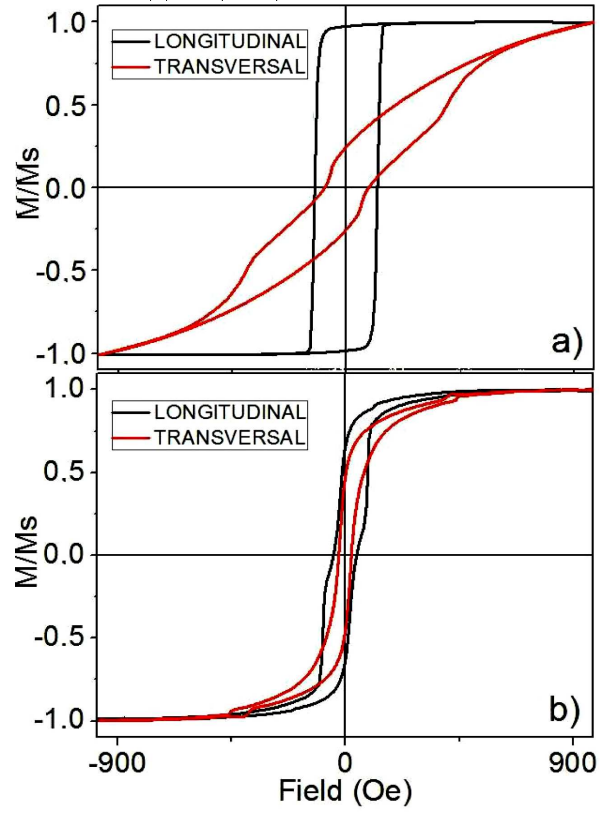

Fig. 2. Step-like behavior for (a) Ni-based MPlC DVD $/ / \mathrm{Ni}(100) / \mathrm{SiO}_{2}(10)$ and for (b) $\mathrm{Ag}-\mathrm{Ni} \mathrm{MPlC}$ on DVD substrate - DVD $/ / \operatorname{Ag}(80) / \mathrm{Ni}(10)$.

Samples saturate faster and the slope of the hysteresis loops is steeper for all fabricated samples along the easy axis. Induced anisotropy is typical for samples with stripe-like periodic structure and the directions of the easy and hard axes are determined by the direction of the stripes. Magnetic moment of every system strives to be into the state with a minimal energy. Any interfaces cause appearance of the stray fields which increase the value of the potential energy. In our case such interfaces are between the ferromagnetic material and air or $\mathrm{SiO}_{2}$. Therefore, the magnetic moment of the clusters during the deposition process orients along the stripes.

The second result was in observation of a step-like behavior of the hysteresis loops in dependence of the magnetic moment versus magnetic field. For the Ni-based MPICs on DVD substrate with thin ferromagnetic layer step-like behavior of the hysteresis loops was observed in the direction perpendicular to the direction of the stripes on the DVD substrate. For the Ni-based MPlCs on DVD substrate with thick ferromagnetic layer a step-like behavior was observed in the direction parallel to the di- 
rection of the stripes. This fact requires further investigation which will be held in nearest future.

Comparison of the slopes of dependences of the magnetic moment on magnetic field in case of transverse plasmon propagation way is shown in Fig. 2. It can be noticed that anisotropy of the Ni-based MPlC with thick ferromagnetic layer is stronger than for the same structure with thin ferromagnetic layer. It can be mentioned that the value of the coercive force grows with increase of the thickness of the ferromagnetic layer.

We analyzed possible reasons of the appearance of such behavior in dependence of the magnetic moment versus magnetic field for DVD- and BD-based structures and decided to consider substrates more thoroughly. Spatial profiles of the samples obtained by AFM are shown in Fig. 3 and schematic structure of the substrate stripes is shown in Fig. 4.

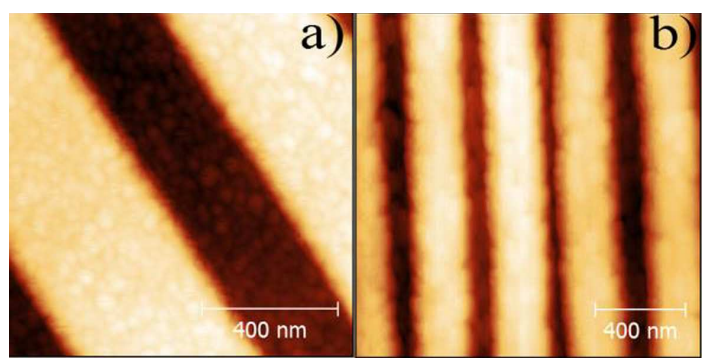

Fig. 3. AFM images of MPlCs based on substrates made of (a) DVD and (b) BD. Brighter areas correspond to stripes.

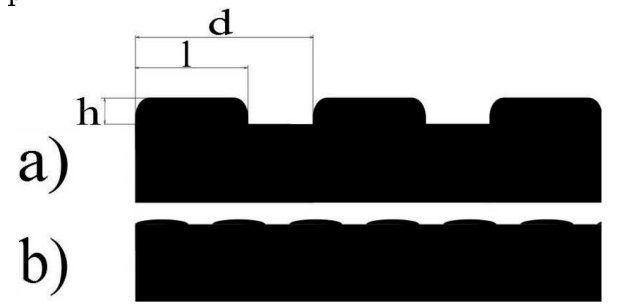

Fig. 4. Schematic structure of substrate stripes for substrates made of (a) DVD and (b) BD digital discs. Here $d$ is period of a grating, $l$ is stripe width and $h$ is stripe height.

Stripes on the surface of the substrate made of DVD digital disk have shape similar to meander. Spatial profile of the polymer spiral gratings inside the DVD disc obtained by AFM showed that period of the grating $d$ is equal to $740 \pm 50 \mathrm{~nm}$, track width $l$ is approximately equal to $470 \pm 50 \mathrm{~nm}$ and stripe height $h$ is approximately equal to $\approx 100 \mathrm{~nm}$. Sputtered on DVD substrate ferromagnetic material layer will cover top and lateral sides of the substrates stripes and gaps between them. The step-like hysteresis loops in DVD-based MPlCs can be explained by magnetostatic interaction between the parts of Ni films that covered the substrate in non-uniform way.

Spatial profile of the polymer spiral gratings inside the BD digital disc has such parameters: $d$ is equal to $320 \pm 20 \mathrm{~nm}, l$ is equal to $220 \pm 15 \mathrm{~nm}$ and profile height $h$ is approximately equal to $\approx 20 \mathrm{~nm}$. Stripes have quasisinusoidal shape without abrupt changes of relief and height differences. Consequently even a very thin layer of the ferromagnetic material should cover the substrate as a uniform layer.

\section{Conclusions}

We have studied MPlCs with different thicknesses of the ferromagnetic layer and spatial profiles of substrates and found that all of fabricated MPlCs have induced anisotropy that can be explained by stripe-like periodic structure of their substrates. Also we found step-like behavior of the hysteresis loops in the direction along the substrate stripes for the Ni-based MPlCs on substrates made of DVD digital discs that can be explained by the interaction between $\mathrm{Ni}$ films on the top and the lateral sides of the substrate stripes.

In conclusion: the thicker the ferromagnetic layer, the more pronounced a step in the dependence of the magnetic moment versus magnetic field and the higher value of the coercive force.

\section{Acknowledgments}

We want to thank User Facilities Center "Innovation Park" for making possible fabrication of our samples.

The work was carried out with financial support from the Ministry of Education and Science of the Russian Federation in the framework of Increase Competitiveness Program of MISiS and in the framework of government assignment (No. 3.2582.2014/K). K. Chichay greatly acknowledges grant RFBR, project No. 14-0231850. V. Belyaev and A. Fedyanin acknowledge RFBR grant No. 14-32-50951. V. Rodionova acknowledges RFBR grant No. 14-32-50920.

\section{References}

[1] J. Homola, Chem. Rev. 108, 462 (2008).

[2] P. Ghenuche, R. Quidant, Phys. Rev. Lett. 101, 116805 (2008).

[3] V. Temnov, Nature Photon. 6, 728 (2012).

[4] A.B. Khanikaev, A.B. Baryshev, P.B. Lim, H. Uchida, M. Inoue, A.G. Zhdanov, A.A. Fedyanin, A.I. Maydykovskiy, O.A. Aktsipetrov, Phys. Rev. B 78, 193102 (2008).

[5] A.A. Grunin, A.G. Zhdanov, A.A. Ezhov, E.A. Ganshina, A.A. Fedyanin, Appl. Phys. Lett. 97, 261908 (2010).

[6] C. Clavero, K. Yang, J.R. Skuza, R.A. Lukaszew, Opt. Expr. 18, 7743 (2010).

[7] V.I. Belotelov, I.A. Akimov, M. Pohl, V.A. Kotov, S. Kasture, A.S. Vengurlekar, A.V. Gopal, D.R. Yakovlev, A.K. Zvezdin, M. Bayer, Nat. Nanotechnol. 6, 370 (2011).

[8] A.A. Grunin, N.A. Sapoletova, K.S. Napolskii, A.A. Eliseev, A.A. Fedyanin, J. Appl. Phys. 111, 07A948 (2012).

[9] A.A. Grunin, A.V. Chetvertukhin, T.V. Dolgova, A.A. Ezhov, A.A. Fedyanin, J. Appl. Phys. 113, 17A946 (2013).

[10] C. Roberts, K. Gordon, in: Encyclopedia of Biophysics, Springer, Berlin 2013, p. 79.

[11] Q. Wang, W. Moerner, Nature Meth. 11, 555 (2014). 